\title{
Dielectric investigation of the temperature dependence of the nonexponentiality of the dynamics of polymer melts
}

\author{
A. Alegría and J. Colmenero \\ Departamento de Física de Materiales y Centro Mixto CSIC-UPV/EHU, Facultad de Química, UPV/EHU, \\ Apartado 1072, 20080 San Sebastián, Spain \\ P. O. Mari and I. A. Campbell \\ Laboratoire de Physique des Solides, Université Paris-Sud, 91405 Orsay, France \\ (Received 9 October 1998; revised manuscript received 24 December 1998)
}

\begin{abstract}
Using broad band dielectric spectroscopy $\left(10^{-5}-10^{9} \mathrm{~Hz}\right)$, combining time domain and frequency domain techniques, we study the temperature dependence of the non-Debye character of the $\alpha$ relaxation of polymer melts in the glass transition temperature $T_{\mathrm{g}}$ range. The $\alpha$ relaxation process is described in terms of the Kohlrausch-Williams-Watts relaxation function which has a single parameter $\beta$ to characterize the nonexponentiality of the relaxation. At high temperatures, $\beta$ remains nearly insensitive to temperature changes, whereas in the vicinity of $T_{\mathrm{g}}$ a nearly linear increasing of $\beta$ with temperature is found. The temperature range where the change of the $\beta(T)$ behavior occurs is located for all the polymers investigated around $1.2 T_{g}$. Moreover, our results indicate a common value of $\beta \approx 1 / 3$ at the temperature where the relaxation time diverges. The $\beta(T)$ behavior near $T_{g}$ is discussed in terms of a "rugged landscape" phase space which allows us to rationalize both the $\beta(T)$ behavior observed as well as the similarities of our findings near $T_{g}$ with the results reported in simulations on Ising spin glasses and other model systems. [S1063-651X(99)04306-8]
\end{abstract}

PACS number(s): 64.70.Pf, 77.22.Gm, 61.41.+e

\section{INTRODUCTION}

The dynamics of glass-forming materials in the glasstransition range manifests, in relaxation techniques, as the main relaxation process which is commonly referred to as $\alpha$ relaxation. The $\alpha$ relaxation presents a series of universal features which are observed in most of the glass-forming liquids independently of the different particularities at the microscopical level. The main universal features are a nonexponential behavior of the relaxation function (see Ref. [1] for an excellent review of stretched exponential behavior) and a temperature dependence of the relaxation rate which does not follow an Arrhenius-law but seems to diverge some ten degrees below the experimentally accessible temperature range [2]. The former of these features is usually well accounted by the stretched exponential relaxation function, which was introduced heuristically more than 150 years ago by Kohlrausch [3] and afterwards applied to the dielectric relaxation by Williams and Watts [4]. The KohlrauschWilliams-Watts (KWW) equation reads as

$$
\phi(t)=\exp \left[-(t / \tau)^{\beta}\right]
$$

where $\tau$ is a characteristic time scale and $\beta$ the nonexponentiality parameter. It is worth emphasizing that the $\mathrm{KWW}$ equation sometimes is used to represent an actual microscopic correlation function as, for example, in neutron scattering (the density-density correlation function), in ${ }^{13} \mathrm{C}$ nuclear magnetic resonance (the carbon-proton bond orientation correlation function), or in numerical simulations, while in other cases it represents a macroscopic correlation function or the response of a given system to a steplike excitation.

In glass formers systems the non-Arrhenius temperature dependence of $\tau$ is found to be well parametrized by means of the empirical Vogel-Fulcher equation [5]

$$
\tau=\tau_{\infty} \exp \left(\frac{B}{T-T_{0}}\right),
$$

where $T_{0}$ is the temperature where the relaxation time seems to diverge. Concerning the temperature dependence of $\beta$, the situation is not clear so far. A reason for this would be that different kinds of behavior have been reported depending on the system investigated and the experimental technique used. What seems to be rather general is that in polymers the temperature dependence of $\beta$ is usually weak. This, in combination with the very strong temperature dependence of the relaxation time close to the glass transition temperature $T_{g}$ makes it very difficult to determine accurately the temperature dependence of $\beta$ when experimental techniques with narrow dynamical range are used. As an example, by using a technique able to cover 4 decades around a time scale of $1 \mathrm{~s}$ the typical temperature range accessible is of about $20 \mathrm{~K}$. This situation is common in mechanical relaxation measurements where the time temperature superposition is often found to apply [6], i.e., the possible temperature dependence of $\beta$ is hardly detectable. In spite of that, some general trends have been established. For low molecular weight (LMW) liquids $\beta(T)$ seems to approach unity at high temperatures, i.e., the exponential decay corresponding to the Debye relaxation is expected in the very high temperature limit. On the contrary, a Debye-like relaxation is hardly found for the segmental dynamics of polymers and instead a value of $\beta$ around 0.5 is typically observed at high temperatures [7]. On the other hand, compilations of mechanical relaxation results for many amorphous linear polymers [8] show $\beta$ values at 
TABLE I. Values of several quantities and fitting parameters describing the behavior of the polymers investigated.

\begin{tabular}{|c|c|c|c|c|c|c|c|c|c|}
\hline POLYMER & $T_{g}(\mathrm{~K})$ & $\sigma\left(T_{g}\right) \quad\left(\Omega^{-1} \mathrm{~m}^{-1}\right)$ & $\Delta \epsilon\left(T_{g}\right)$ & $\tau_{\infty}(\mathrm{ps})$ & $\mathrm{B}(\mathrm{K})$ & $T_{0}(\mathrm{~K})$ & $T_{g D}(\mathrm{~K})$ & $\beta_{\infty}$ & $x_{0}$ \\
\hline Polybutadiene $^{\mathrm{a}}(\mathrm{PB})$ & 238 & & 0.11 & 13.6 & 864 & 200 & 229 & & \\
\hline Poly(vinyl methyl ether) (PVME) & 247 & $4.1 \times 10^{-20}$ & 0.72 & 0.38 & 1304 & 205 & 243 & 0.52 & 0.15 \\
\hline Poly(vinyl ethylene) (PVE) & 272 & & 0.12 & 10.4 & 747 & 245 & 269 & 0.59 & 0.25 \\
\hline Poly(vinyl acetate) (PVAc) & 315 & $2.4 \times 10^{-15}$ & 4.4 & 0.84 & 1410 & 267 & 311 & 0.67 & 0.11 \\
\hline Phenoxy $^{\mathrm{b}}(\mathrm{PH})$ & 370 & $5.5 \times 10^{-16}$ & 0.50 & 22 & 592 & 349 & 369 & & \\
\hline Polysulfone (PSF) & 463 & $1.3 \times 10^{-14}$ & 3.5 & 14 & 729 & 436 & 457 & 0.99 & 0.24 \\
\hline
\end{tabular}

${ }^{\mathrm{a}} 68 \% 1,2$ units and $32 \% 1,4$ units.

${ }^{\mathrm{b}} \mathrm{Poly}(2$-hydroxy propyl ether of bisphenol-A).

$T_{g}$ close to 0.4 , whereas for LMW liquids the typical values at $T_{g}$ are around or higher than 0.5 [1].

Most of the relaxation techniques used in the past were restricted to a relatively narrow frequency window. However, during the last years, some experimental techniques, such as dielectric relaxation, nuclear magnetic resonance, and light scattering, among others, became able to cover a very broad spectral range, which nowadays allows one to investigate in detail the temperature behavior of $\beta$. In particular, dielectric relaxation is able to cover up to 10 decades in time scale using a single spectrometer and more than 14 decades by combining different setups. In addition, the dielectric relaxation techniques are very sensitive allowing very accurate measurements. Taking advantage of these features, we have performed a detailed study of the temperature dependence of $\beta$ in the segmental dynamics range of several polymers using broad band dielectric spectroscopy over 14 orders of magnitude in the time scale. To get this extremely broad dynamical range, we have combined time and frequency domain techniques. The corresponding temperature range is of about $150 \mathrm{~K}$. The polymers investigated have very different chemical structures of the monomeric units, average molecular weight, and polydispersity. They include commercial and tailor-made samples and their values of $T_{g}$ spread out over more than $200 \mathrm{~K}$.

The article is organized as follows: In Sec. II detailed information about the samples and the experimental techniques used is given. In Sec. III we explain the procedure followed for the data analysis and we show the results obtained. In Sec. IV a discussion of our findings in the framework of the current ideas on the dynamics of glass-forming system is depicted. Moreover a comparison between the experimental behavior found in polymers and the results previously reported on model systems, including simulations on Ising spin glasses, is presented. Finally in Sec. V the main conclusions of the present work are drawn.

\section{EXPERIMENTAL}

\section{A. Samples}

The samples investigated, which are listed in Table I, are well known amorphous polymers most of then commercially available. They present a quite different chemical structure from the monomeric unit. The glass transition temperatures of the polymers were determined as the midpoint of the heat capacity increment as measured by using a differential scanning calorimetric (DSC) at $10 \mathrm{~K} / \mathrm{min}$ on samples previously cooled from above $T_{g}$ at the same rate. The values obtained are listed in Table I and spread out over about $230 \mathrm{~K}$ according with the different chain stiffness. The samples also differ in the strength and location of the dipole moment of the monomeric units. However, due to the randomness in the dipole moment orientation along the chain contour, only the reorientation of the component perpendicular to the backbone chain manifests itself as a dielectric relaxation, i.e., no dielectric relaxation associated with the fluctuation of the end-to-end chain distance is present in any of the selected polymers. Furthermore, these polymers have the advantage of showing the $\alpha$ relaxation well separated from other processes such as the Johari-Goldstein secondary relaxation [9], which are, in the temperature range investigated, much weaker than the main relaxation process.

The samples for dielectric experiments were prepared by melting the "as received" product directly on gold-plated stainless steel electrodes in a vacuum furnace at $T_{g}+100 \mathrm{~K}$. After degassing the sample, the upper electrode was placed on the sample with a light pressure. A fixed distance between the electrodes was maintained by means of Teflon spacers. The sample with the electrodes was held under vacuum during the cooling process down to room temperature.

\section{B. Frequency domain dielectric spectroscopy}

The dielectric measurements in the frequency domain and in the range from $10^{-2}-10^{9} \mathrm{~Hz}$ were performed following standard procedures. The experimental setup covering the range $10^{-2}-10^{7} \mathrm{~Hz}$ consisted of a Solarton-Schlumberger frequency response analyzer SI 1260, which was supplemented by using a high impedance preamplifier of variable gain. The resolution in the loss factor was $10^{-4}$ in this case. For the frequency range $10^{6}-10^{9} \mathrm{~Hz}$ a Hewlett-Packard impedance analyzer HP4191A was used which allowed a resolution in the loss factor of $10^{-2}$. Frequency scans were performed at constant temperature, which was decreased in steps. Both equipments were supplied by Novocontrol $\mathrm{GmbH}$ with a temperature control system which allowed a stability better than $0.1 \mathrm{~K}$.

\section{Time domain dielectric measurements}

The setup used for these measurements has been described elsewhere $[10,11]$. It is formed by a KEPCO APH 2000 M power supply, which provides a constant de voltage set to $200 \mathrm{~V}$, and a KEITHLEY 642 electrometer with an intensity resolution of $10^{-17} \mathrm{~A}$. The sample cell consists in two aluminum electrodes isolated with Teflon in the upper 
part of the cell, so the Teflon insulator is held at room temperature. A CERBERE temperature controller provides a temperature stability better than $0.1 \mathrm{~K}$. The same sample used for the low frequency measurements was placed in the cell. The experiments were performed isothermally by polarizing the sample capacitor during a time $t_{p}$ and, immediately, after removing the poling field, measuring the subsequent depolarization current. Under these conditions, and on the basis of the superposition principle, the measured depolarization current can be expressed as a function of the relaxation function $\phi(t)$ as $[10-12]$

$$
I(t) \propto \frac{d}{d t}\left[\phi(t)-\phi\left(t+t_{p}\right)\right] .
$$

Note that the relaxation function $\phi(t)$ in Eq. (3) represents the decay of the polarization of a fully polarized sample after removing the electric poling field, i.e., the response to a steplike excitation, which would correspond to the autocorrelation function of the total electric moment. For polarization times $t_{p}$ much larger than the characteristic relaxation time, from Eq. (3) it follows that the measured current is proportional to the first derivative of the relaxation function. This experimental procedure is suitable in the temperature range where the depolarization process is slower than the characteristic time of the electrometer response, about $0.1 \mathrm{~s}$, but is sufficiently fast to provide measurable values of the depolarization current. These limitations allowed us to obtain the dielectric response at the temperatures where the relaxation time is in the range $1 \mathrm{~s}<t<10^{5} \mathrm{~s}$. Great care was taken to ensure that, for each measurement, the sample was in a complete equilibrium before and during the run. The equilibrium situation was taken as that for which a further enlargement of the annealing times does not modify the relaxation behavior. Close, but below, the glass transition temperature region this implies annealing times of days [11].

\section{RESULTS}

The dielectric relaxation corresponding to the segmental dynamics of the polymers investigated is the main relaxation process in the temperature range investigated. Some representative dielectric loss patterns, $\epsilon^{\prime \prime}(\omega)$, are shown in Fig. 1. In addition of the dielectric loss peak, in some polymers, an extra contribution is apparent at low frequencies. In these cases the dielectric losses increase at low frequencies as $\omega^{-1}$ indicating that the origin of such a contribution is the dc conductivity, $\sigma$. The estimated values of $\sigma\left(T_{g}\right)$ for the polymers investigated are shown in Table I. Note that for PVE and $\mathrm{PB}$ the conductivity was under the detection level of the equipment used. It is also worthy of remark that for PSF, the high conductivity contribution precluded the accurate dielectric measurements in the time domain. On the other hand, for $\mathrm{PH}$ and $\mathrm{PB}$, the weakness of the dielectric $\alpha$ relaxation makes the measurement in the high frequency range subjected to very large uncertainties and therefore have not been used in the present analysis.

The frequency dependence of the complex permittivity $\epsilon^{*}=\epsilon^{\prime}-i \epsilon^{\prime \prime}$ is related with the relaxation function as [12]

$$
\epsilon^{*}(\omega)=\epsilon_{\infty}+\Delta \epsilon \int_{0}^{\infty}\left[-\frac{d \phi(t)}{d t} \exp (-i \omega t)\right] d t-i \frac{\sigma}{\omega},
$$

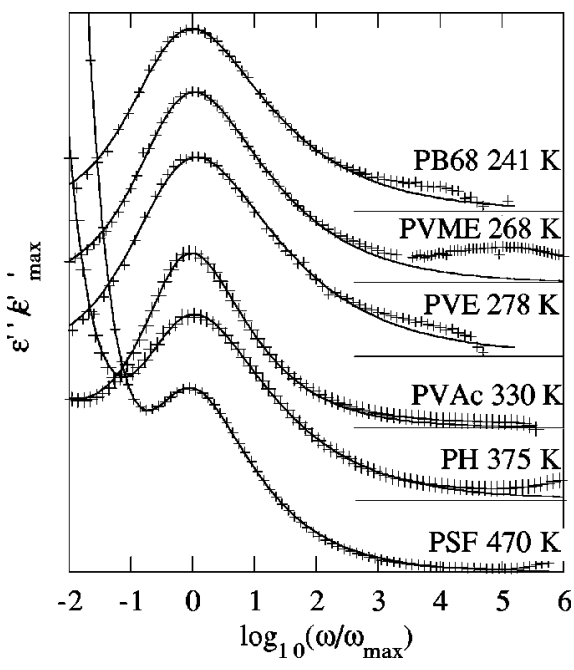

FIG. 1. Normalized dielectric losses corresponding to the segmental dynamics of the polymers investigated. The lines are the fitting curves as described in the text.

where $\epsilon_{\infty}$ is the unrelaxed value of the permittivity and $\Delta \epsilon$ is the relaxation strength. It is worth emphasizing that Eq. (4) is formally correct when $\phi(t)$ represents the response to a steplike excitation. However, under certain conditions (e.g., $\Delta \epsilon$ $\ll 1)$ this stepresponse $\phi(t)$ also represents to a good approximation a microscopic dipolar correlation function (see, for example, p. 159, Vol. II in Ref. [12]). Estimated values of $\Delta \epsilon$ for the polymers investigated are shown in Table I. It can be seen that only PVAc and PSF are far from the condition $\Delta \epsilon \ll 1$. Thus, for these two polymers $\phi(t)$ will not describe a microscopic dipolar correlation function but only the stepresponse.

Although for a KWW relaxation function the integral in Eq. (4) has to be evaluated numerically, it has been shown that in this case, and in the frequency range corresponding to the loss peak, an approximate form of Eq. (4) is given by [13]

$$
\epsilon^{*}(\omega) \approx \epsilon_{\infty}+\frac{\Delta \epsilon}{\left[1+\left(i \omega / \omega_{c}\right)^{\alpha}\right]^{\gamma(\alpha)}}-i \frac{\sigma}{\omega}
$$

with $\gamma(\alpha)=1-0.812(1-\alpha)^{0.387}$. In Eq. (5), which is a particular case of the well known Havriliak-Negami equation [12], $\alpha$ is a shape parameter in the range of zero unity ( 0 $<\alpha \leqslant 1)$ and $\omega_{c}$ is a characteristic frequency of the order of $\tau^{-1}$. The relationship among these parameters and those of the KWW equation are given by [13]

$$
\begin{gathered}
\beta^{1.23}=\alpha \gamma(\alpha) \\
\log _{10}(\tau)=-\log _{10}\left(\omega_{c}\right)-2.6(1-\beta)^{0.5} \exp (-3 \beta) .
\end{gathered}
$$

As we have commented above, most of the polymers present a secondary relaxation which manifests as a weak dispersion process at high frequencies. To minimize the influence of this secondary relaxation in the fitting parameters obtained in the frequency domain, the dielectric losses were fitted using the data points with values above $10 \%$ of the peak value. An example of the quality of such a fitting is shown in Fig. 1. As can be seen, Eq. (5) allows a perfect 


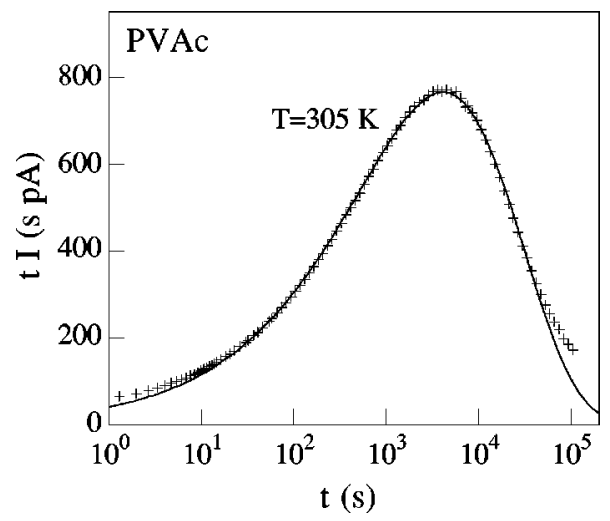

FIG. 2. Typical dielectric relaxation experiment in the time domain. The line is a fitting curve obtained as described in the text.

description of the main part of the dielectric losses in all the samples. For those polymers showing a significant conductivity, the frequency range for fitting was restricted in the low frequencies to the range where the real part of $\epsilon^{*}(\omega)$ is actually frequency independent and therefore the conductivity contribution is well accounted by the corresponding term appearing in Eqs. (4) and (5). In spite of that, the parameter values determined in these cases at low temperatures, where the overlapping between the conductivity tail and the loss peaks is more pronounced, are subjected to higher uncertainties, particularly for PH and PSF.

The depolarization current measured in time domain experiments in the glass transition range resulted very well described by means of Eq. (3) with a KWW relaxation function for $\phi(t)$. A representative experimental curve is shown in Fig. 2. In this figure the quantity $t I$ is plotted as a function of the depolarization time. It follows from Eq. (3) that for experiments where the polarization time is much larger than $\tau$ (as the one presented in Fig. 2) this quantity shows a maximum at time $\tau$. In the figure it is apparent that small discrepancies are present in the limits of very short or very large times. This could be originated by contributions to the measured intensity other than the segmental dynamics (secondary relaxations or interfacial polarization mechanisms). Taking this into account and in order to minimize the effects of these contributions on the fitting parameters obtained in the time domain, also in this case, only the data points with values above $10 \%$ of the peak value were considered in the fitting procedure.

Following the fitting procedures described above the segmental dynamics of the polymers investigated can be characterized in the whole temperature range investigated by only two dynamical parameters, the relaxation time $\tau(T)$ and the nonexponentiality parameter $\beta(T)$. The temperature behavior of $\tau$ shows the typical non-Arrhenius temperature dependence (see Fig. 3) which can be well described by a VF expression [Eq. (2)]. The values of the corresponding VF parameters are shown in Table I. It should be noted that in spite of the extremely large dynamical range investigated the resulting values of the VF temperature are determined with a typical uncertainty of $\pm 5 K$. From these VF descriptions we have determined a dielectric glass transition temperature defined $T_{g D}$, as $\tau\left(T_{g D}\right)=100 \mathrm{~s}$, see Table I. The resulting values are very close to the corresponding calorimetric glass transition temperatures which is a strong evidence that the

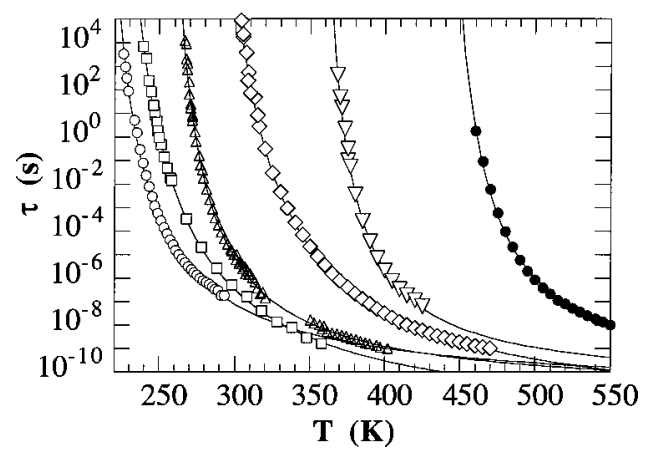

FIG. 3. Temperature dependence of the relaxation time corresponding to the segmental dynamics of the polymers investigated: $\operatorname{PB}(\bigcirc), \operatorname{PVME}(\square), \operatorname{PVE}(\triangle), \operatorname{PVAc}(\diamond), \operatorname{PH}(\nabla)$, and PSF (๑). Lines are fitting curves by using Eq. (2).

dielectric relaxation investigated probes the segmental polymer dynamics.

With respect to the temperature behavior of $\beta$, in Fig. 4 we show the obtained results as a function of $T / T_{g D}$, which facilitates the comparison. Four of the polymers show very similar values of $\beta\left(T_{g D}\right) \approx 0.4$ and eventually a hightemperature limiting value of around 0.5. As mentioned above, these values are typical for the majority of the polymer melts. However, PVAc and PSF show systematically higher values of $\beta$ in the whole temperature range investigated. Nevertheless, it is apparent that for all the polymers $\beta$ increases with $T$ in the range $T<1.23 T_{g D}$ (region I) and reach a nearly constant value in the high-temperature range (region II). The fact that the greater the high-temperature limiting value of $\beta$ is the stronger the temperature dependence of $\beta$ in the $T_{g}$ range yield a convergence in the lowtemperature side of the $\beta(T)$ behaviors of the different polymers. In agreement with that, by extrapolating the linear region close to $T_{g D}$ down-to $T=0.9 T_{g D}$ one obtains values of $\beta$ in the range $0.32-0.38$ (see Fig. 4) for all the polymers. Furthermore, taking into account the uncertainty in the extrapolation, this result would be compatible with a common value of $\beta$ for all the polymers in this temperature range. It is noteworthy that this temperature interval coincides with the

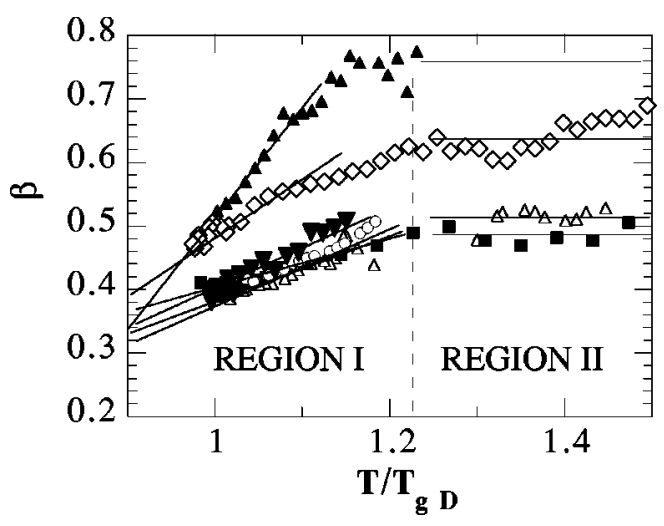

FIG. 4. Temperature dependence of the nonexponentiality of the segmental dynamics of the polymers investigated: PB (circles), PVME (filled squares), PVE (empty triangles), PVAc (empty diamonds), PH (filled inverted triangles), and PSF (filled triangles). The lines in region I are linear fitting of the data in the interval $(1.0,1.1)$ whereas in region II they are to guide the eye. 
region where the relaxation times of the different polymers seem to diverge, the values of $T_{0}$ are in all cases in the range from $0.85 T_{g}$ to $0.95 T_{g}$ (see Table I).

\section{DISCUSSION}

We have shown that the temperature dependence of $\beta$ in polymer melts allows us to distinguish two different regimes, one at high temperature $\left(T>1.23 T_{g}\right)$ where $\beta$ is nearly independent of temperature, region II, and a low-temperature region $\left(T<1.23 T_{g}\right)$ where $\beta$ seems to increase with temperature in a nearly linear way. The existence of two dynamical ranges in supercooled liquids has been discussed for some years (see Ref. [14], and references therein) stimulated by the predictions of the mode coupling theories. Most of the evidence for a change in mechanism in the supercooled liquid dynamics is based on different representations (scaling) of the temperature dependence of the relaxation times and on the differences between the temperature dependencies of the transport properties of a given system. Note that our finding does not concern the $\tau(T)$ behavior, but the temperature dependence of the nonexponentiality of the $\alpha$ relaxation. The interpretation of the two dynamical regimes, although in discussion, is based in the idea that the free diffusion in the high-temperature region is slowed down dramatically when the temperature is lowered and therefore the activated processes become relevant for the dynamics in the low temperature range close $T_{g}$. This point of view is compatible with the energy landscape picture $[14,15]$ in which the potential energy of the system forms a hypersurface. This hypersurface has multitude of relative minima which are separated by widely distributed energy barriers. At high temperatures the dynamics of the liquid is hardly sensitive to the presence of these minima, since the thermal energy is high. Lowering the temperature the thermal energy is diminished and the system starts to "see" the "rough landscape." Therefore, close to $T_{g}$ the point in the phase space representing the instantaneous configuration of the system must follow a path along tortuous valleys of low-energy states while skirting around mountains of high-energy states. For fragile liquids the temperature where the change of mechanism would take place is estimated to be around $1.3 T_{g}[14]$ which is not far from the value of the crossover temperature found for the polymers investigated here (see Fig. 4). Thus, our findings would be the first experimental evidence based on the temperature dependence of the spectral shape of the existence of two dynamical regimes in polymer melts. A comparison with results based on the temperature dependence of relaxation times is not easy since in polymers the different representations used in other glass forming liquids hardly show evidence of these two regions. From our data only in PVAc such representations allow us to envisage two different regions with a crossover temperature around $390 \mathrm{~K}$, i.e., $1.26 T_{g}$.

On the other hand, our findings seem to indicate the possibility of a common value of $\beta$ (not far from $1 / 3$ ) at the temperature $T_{0}$ where the relaxation times would become infinite. It is noteworthy that similar results have been reported for a series of different model systems trying to mimic the glass-forming system behavior. This is the case of the simulation on the dynamics of different Ising spin glasses
(ISGs) [16-23]. In these simulations the magnitude usually obtained is the spin autocorrelation function which is described by some authors in terms of the KWW function. In particular, Ogielski studied a three-dimensional (3D) ISG with random binary near neighbor $\pm J$ interactions [16] and found that although at very high temperatures $\beta$ is close to 1 , when the temperature is lowered the dynamics becomes strongly non-Debye and a linear temperature dependence of $\beta$ is obtained on approaching the freezing temperature. When this linear behavior is extrapolated to the freezing temperature where the relaxation time diverges one obtains $\beta$ $=0.35 \pm 0.02[17,18]$. The results obtained in other simulations on ISG systems very different for the one investigated by Ogielski, namely, on a $p$-spin ISG on a cubic lattice in dimension 3 [19], confirmed a linear temperature dependence of $\beta$ on cooling to the freezing temperature. Moreover, a value of $\beta=0.37$ is found at the lowest temperatures investigated, which is similar to that of most of the polymer melts in the $T_{g}$ range. In addition, simulations carried out for ISGs with $\pm J$ and Gaussian near neighbor interactions in dimensions 3 and 4 , also showed $\beta$ tending to about $1 / 3$ when the sample size (and hence $\tau$ ) is extrapolated to infinity [20]. In these simulations, the temperature was held fixed at the appropriate ordering temperature and the dynamics was studied as a function of sample size. On the other hand, experiments on laboratory "model" systems, for instance, in a polydisperse polymer solution [24] and in a colloid [25] also showed a value of $\beta$ tending to $1 / 3$ when $\tau$ diverges. In these latter measurements the colloid concentration rather than the temperature is the control parameter which is modified. As the concentration is varied progressively from a low value to the critical concentration at which the colloid gels, the relaxation time increases and tends to diverge. The stretched exponential exponent $\beta$ changes from 1 to a value very close to $1 / 3$ (see Fig. 4 in Ref. [25]). Finally, very recent simulations on other model systems very different from the ISGs also show a similar tendency [26].

From the previous discussion, it is apparent that, when the relaxation time tends to diverge, there exist remarkable similarities in the $\beta(T)$ behavior of the polymer melts on one hand and the model systems on the other hand. In addition, some similarities in the experimental dielectric dynamical susceptibility of low molecular weight glass formers and spin glasses has been also suggested [27,28]. These remarkable similarities between the different families of systems can be quantitatively checked if $\beta$ is plotted as a function of a new dimension-less variable $x$ which depends on the relaxation time $\tau$, with $T$ as an implicit parameter (see Fig. 5). The variable $x$ is defined as

$$
x=\frac{1}{\log _{10}[\tau(T) / \Lambda]} .
$$

In Eq. (8), which yields $x=0$ for diverging times, $\Lambda$ is a parameter defining the time units used. Thus, Eq. (8) allows us to compare results obtained in very different systems if the appropriate time units are used. For the polymers we have taken $\Lambda$ as the inverse of a typical phonon frequency $\left(\Lambda=10^{-12} \mathrm{~s}\right)$. A different choice $\left(10^{-11}\right.$ or $10^{-13} \mathrm{~s}$, for instance) only dilates or contracts the $x$ axis, particularly for the larger values of $x$, but does not affect the general appear- 

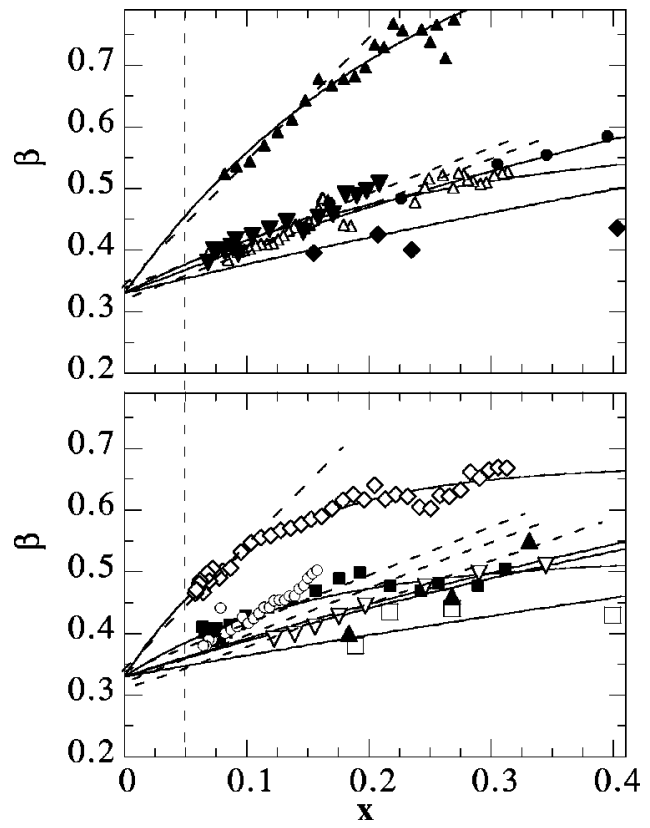

FIG. 5. Comparison between the nonexponentiality of the segmental dynamics of the polymers investigated: PB (circles), PVME (filled squares), PVE (empty triangles), PVAc (empty diamonds), PH (filled inverted triangles), and PSF (filled triangles), and that of the relaxation of model systems: 3D ISG model with bimodal interaction distribution (empty inverted triangles) [16], 3D four-spin ISG model (filled circles) [23], 3D Gaussian ISG model (empty squares) [20], 3D $\pm J$ ISG model (filled triangles) [20], and colloids (filled diamonds) [25]. The $x$ axis is defined as $1 / \log _{10}[\tau(T) / \Lambda]$ (see the text). The dotted lines correspond to possible linear extrapolations. The solid lines are fitting curves obtained by using Eq. (9). The vertical dashed line represents relaxation times of 3 years, so the sector to the left of these lines is essentially inaccessible.

ance of the plot. The value of $\Lambda$ used for the ISGs simulations is the "'attempt time,", which is 1 Monte Carlo Step per spin (MCS), whereas for the colloid systems $\Lambda$ was taken as the value of $\tau$ in the limit of zero concentration.

In this representation a comparison of the behavior of the nonexponentiality parameter $\beta$ in polymers and in the model systems is straightforward. Moreover, this type of plot brings out the fact that further measurements, or numerical simulations, at lower temperatures to push the limit closer to $x$ $=0$ rapidly become totally impractical. Thus extending a plot down to $x=0.05$ would require studying relaxation at temperatures corresponding to $\tau$ values extended up to 3 years in experiments and up to $10^{20} \mathrm{MCS}$ in the simulation. Therefore the results shown in Fig. 5 can be taken to cover almost the entire range for $\beta(x)$ that can be attained with present day means. It can be seen that each of the sets of data taken in the accessible domain for the different systems are compatible (within the usual experimental uncertainties) with $\beta$ varying fairly linearly with $x$ as $x \rightarrow 0$. However, whereas for the model systems the linear behavior extends on a wide range $(0<x<0.5)$ for polymers it is restricted only to low values of $x$. The approximately linear $\beta(x)$ behavior for the low $x$ values is directly connected with both, the linear dependence of $\beta(T)$ close to $T_{g}$ and the VF dependence of $\tau(T)$ with a pre-exponential factor of the order of $\Lambda$. These two conditions are approximately verified for the polymers investigated. A linear extrapolation of the $\beta(x)$ data to $x=0$ (see dotted lines in Fig. 5) shows a $\beta(x)$ value tending to near $1 / 3$ at the critical temperature at which $\tau$ diverges $(x=0)$. Other extrapolations are clearly possible but the limiting $\beta$ values certainly tend to cluster closely. An ad hoc expression for $\beta(x)$ that has the property $\beta(0)$ $=1 / 3$ is

$$
\beta(x)=\beta_{\infty}\left[1-\left(1-\frac{1}{3 \beta_{\infty}}\right) \exp \left(-\frac{x}{x_{0}}\right)\right],
$$

where the fitting parameters for each curve are $\beta_{\infty}$, which is determined by the high-temperature limiting value and $x_{0}$ which indicates the crossover region. In Fig. 5 we show the fitting curves obtained using Eq. (9) for those polymers in which the high-temperature and frequency range was accessible. The fitting parameters so obtained are shown in Table I. The same equation also allows to fit the $\beta(x)$ results obtained for model systems but the value of $\beta_{\infty}$ is now unity. As can be seen Eq. (9) gives a satisfactory description for the $\beta(x)$ behavior in the whole range investigated for both polymers an model systems. However, it is found that whereas for polymers the value of $x_{0}$ obtained are in a rather narrow range between 0.11 and 0.25 , for the model systems $x_{0}$ is much larger (1.0-2.0). This would imply that for polymers the $\beta(x)$ behavior would mainly be determined by the value of $\beta_{\infty}$. It is reasonable to consider that the value of $\beta_{\infty}$ should be related to the details of the dynamics of each material. In particular, a limiting value of $\beta_{\infty}$ lower than unity would indicate that, even at high temperatures, there exist some kind of correlation in the reorientation of the dipolar units. This is expected in polymers since the connectivity of the main chain is always present. However, the correlation in the dipole moment orientations for PSF seems to be negligible at high temperatures $\left(\beta_{\infty} \approx 1\right)$, which could be attributed to the fact that in this polymer the main dipolar units $\left(\mathrm{SO}_{2}\right)$ in the backbone chain are well separated (there are four phenyl rings between each couple of dipoles). Nevertheless, other factors as the coupling between the dipolar motions and the segmental dynamics would also determine the value of $\beta_{\infty}$.

On the other hand, since the $\beta_{\infty}$ values vary from one polymer to another, the $\beta$ values splay out when $x$ increases ( $\tau$ decreases). As a result, at $T_{g D}$ (where $\tau=100 \mathrm{~s}$ and $x$ $=0.071)$, there is a dispersion of $\beta\left(T_{g} D\right)$ from about 0.36 to 0.56 (compare with the values given in Ref. [8]). The value of $\beta$ at $T_{g}$ has been widely used as a phenomenological parameter to classify the glass forming systems. For example, on the basis of a trapping model it has been suggested that the Kohlrausch exponent should tend to 0.43 at $T_{g}$ in polymer melts [1]. On the other hand the value of $\beta\left(T_{g}\right)$ has often been related with the so-called fragility of the glass forming systems [29]. The polymers studied here have different fragility (see Fig. 3 and Table I), being in all cases rather fragile systems, but there is not a direct correlation with the corresponding values of $\beta\left(T_{g}\right)$. PH the most fragile and PVME the least fragile of the polymers investigated show a very similar $\beta(T)$ behavior, whereas PVAc, having a fragility similar to PVME, presents a quite different $\beta(T)$ behavior. Therefore, no one of these ideas seems to be supported by the present results. Contrary to these possible cor- 
relations, our finding indicates that the value of $\beta\left(T_{g}\right)$ is mainly determined by the $\beta_{\infty}$ values whereas the lowtemperature limit would be near $1 / 3$ for all the samples. Moreover, the same $\beta(T)$ behavior is found in different families of systems when the time scale divergence is approached as shown in Fig. 5. Because of fundamental restrictions we are obliged to extrapolate the behavior in the very long relaxation time regime, but Fig. 5 suggests that the $\beta$ data are compatible with a clustering around $1 / 3$ in the infinite $\tau$ limit. Because of fundamental equilibration time limitations, there is no way in which it can be proved rigorously that $\beta$ tends to exactly $1 / 3$, but reasonable extrapolations for the data on the six polymers and on the model systems all lie in the range $0.35 \pm 0.05$.

As discussed above, the low temperature dynamics of the polymer melts can be understood in terms of an energy landscape picture. Moreover, it has been recently shown that the energy landscape picture can account quantitatively for several controversial aspects of the $\alpha$ relaxation in supercooled liquids as the glass transition temperature is approached [30]. On the other hand, the landscape picture is widely used in the ISG community. In particular, it was conjectured [31] that dilute randomly distributed sites on a hypercube lead to an extreme example of a rugged landscape and provide a purely geometrical model mimicking the true ISG phase space and its evolution with temperature. Moreover, it was demonstrated numerically [32] that a random walk on the spanning cluster which exists in this model above the "percolation transition" produces a stretched exponential decay of the memory function, with an exponent $\beta$ tending to precisely $1 / 3$ at the critical concentration of sites where in the large system limit the characteristic time diverges. The analogy between on the one hand a spanning cluster which breaks up into small clusters at the percolation transition in the hypercube, and on the other hand a thermodynamic transition as a function of temperature in an ISG system is discussed in Ref. [32]. The fact that, to within practical limitations a similar relaxation behavior is observed in the hypercube model, in the model systems and in the segmental dynamics of polymer melts implies that the phase spaces of these different systems necessarily must have morphologies which resemble each other when their respective transitions are approached. Nevertheless, since at higher temperatures the behavior of the different systems is different (in the model systems a value of $\beta$ tending to 1 is expected whereas for polymers this limiting value is hardly found) the comparison in terms of the landscape morphologies should be restricted to the temperature range where the time scales clearly tend to diverge.

\section{CONCLUSIONS}

We have shown that the segmental dynamics of polymer melts as observed by dielectric relaxation spectroscopy can be well characterized by means of a KWW equation. This description is valid in the whole temperature range investigated, however, the accuracy of this description is limited for some polymers in the low-temperature side by the overlapping of the sample conductivity. $\tau(T)$ follows a VF law and $\beta(T)$ shows a crossover from a high-temperature region where it is essentially temperature independent and a lowtemperature region where $\beta(T)$ increases with temperature. In the low-temperature region, the values of $\beta$ in all the polymers investigated show a tendency to converge in a value near $1 / 3$ at the temperature where $\tau$ diverges. The two regions in $\beta(T)$ roughly coincide with the two dynamical regimes reported for supercooled liquids, which are also expected in the framework of the energy landscape picture. The same framework allows to rationalize the existence of remarkable similarities between the dynamics of both polymer melts and model systems, as their respective time scales diverge. Particularly, a limiting value of $\beta \approx 1 / 3$ at the temperature where the relaxation time would diverge seems to be ubiquitous in these very different families. It is noteworthy, however, that whereas for simulations $\phi(t)$ represents a microscopic correlation function, for dielectric experiments this is not always the case, as it has been discussed above. Since the model systems, and in particular the ISGs, present a well defined critical temperature at which the relaxation time becomes infinity, the similarities found would indicate the possibility of the existence, in polymers, of a real thermodynamic transition at about $T_{0}$, which is inaccessible experimentally, because, due to the time scale divergence, the system cannot attain equilibrium for temperatures more than a few degrees below $T_{g}$.

\section{ACKNOWLEDGMENTS}

A.A. and J.C. acknowledge the support given by Acciones Integradas HF1998-0151, and the following projects: DGICYT Project Nos. PB94-0468 and PB97-0638, GV Project No. EX1998-23 and UPV/EHU Project No. 206.215-G20/98. The support from the "Donostia International Physics Center" is also acknowledged. The simulations were carried out thanks to time allocations from IDRIS (Institut du Développement des Ressources en Informatique Scientifique).
[1] J.C. Phillips Rep. Prog. Phys. 59, 1133 (1996).

[2] F. Stickel, E.W. Fischer, and R. Richert, J. Chem. Phys. 102, 6251 (1995); 104, 2043 (1996).

[3] R. Kohlrausch Pogg, Ann. Phys. (Leipzig) 91, 179 (1854).

[4] G. Williams and D.C. Watts, Trans. Faraday Soc. 66, 80 (1970).
[5] H. Vogel, Z. Phys. 22, 645 (1961); G.S. Fulcher, J. Am. Ceram. Soc. 8, 339 (1925).

[6] N.G. McCrum, B.E. Read, and G. Williams, Anelastic and Dielectric Effects in Polymeric Solids (Wiley, London, 1967).

[7] A. Arbe, D. Richter, J. Colmenero, and B. Farago, Phys. Rev. E 54, 3853 (1996). 
[8] D.J. Plazek and K.L. Ngai, Macromolecules 24, 1222 (1991); R. Böhmer, K.L. Ngai, C.A. Angell, and D.J. Plazek, J. Chem. Phys. 99, 4201 (1993).

[9] G.P. Johari and M. Goldstein, J. Chem. Phys. 53, 2372 (1970).

[10] A. Alegría, E. Guerrica-Echeverría, L. Goitiandía, I. Tellería, and J. Colmenero, Macromolecules 28, 1516 (1995).

[11] A. Alegría, L. Goitiandía, I. Tellería, and J. Colmenero, Macromolecules 30, 3881 (1997).

[12] C.J.F. Böttcher; O.C. van Belle, J. Bordewijk, and A. Rip, Theory of Electric Polarization (Elsevier Scientific, Amsterdam, 1973).

[13] F. Alvarez, A. Alegría, and J. Colmenero, Phys. Rev. B 44, 7306 (1991); 47, 125 (1993).

[14] C.A. Angell, in Complex Behaviour of Glassy Systems, edited by M. Rubi and C. Pérez-Vicente, Vol. 402 of Lecture Notes in Physics (Springer, Heidelberg, 1997), p. 1.

[15] M. Buchanan, Nature (London) 382, 302 (1996).

[16] A.T. Ogielski, Phys. Rev. B 32, 7384 (1985).

[17] N. Kawashima and A.P. Young, Phys. Rev. B 53, R484 (1996).

[18] L.W. Bernardi, S. Prakash, and I.A. Campbell, Phys. Rev. Lett. 77, 2798 (1996).

[19] D. Alvarez, S. Franz, and F. Ritort, Phys. Rev. B 54, 9756 (1996).

[20] I.A. Campbell and L. Bernardi, Phys. Rev. B 49, 728 (1994).

[21] L. Angelani, G. Parisi, G. Ruoco, and G. Viliani, Phys. Rev. Lett. 81, 4648 (1998).

[22] L.W. Bernardi, N. Lemke, P.O. Mari, I.A. Campbell, A. Ale- gría, and J. Colmenero, Physica A 257, 21 (1998).

[23] P.O. Mari and I.A. Campbell, Phys. Rev. E 59, 2653 (1999).

[24] M. Adam, M. Delsanti, J.P. Munch, and D. Durand, Phys. Rev. Lett. 61, 706 (1988).

[25] E. Bartsch, M. Antonietti, W. Schupp, and H. Sillescu, J. Chem. Phys. 97, 3950 (1992).

[26] I.S. Graham, L. Piché, and M. Grant, Phys. Rev. E 55, 2132 (1997).

[27] P.K. Dixon, L. Wu, S.R. Nagel, B.D. Williamson, and J.P. Carini, Phys. Rev. Lett. 65, 1108 (1990); 66, 960 (1991); D. Bitko, S.N. Coppersmith, R.L. Leheny, N. Menon, S.R. Nagel, and T.F. Rosenbaum, J. Res. Natl. Bur. Stand. 102, 207 (1997).

[28] M. Cutroni, A. Mandanici, and A. Piccolo, J. Phys.: Condens. Matter 7, 6781 (1995).

[29] The fragility is defined by Angell [C.A. Angell, J. Non-Cryst. Solids 131-133, 13 (1991)] as an exponent in the VogelFulcher expression for the viscosity $\eta \propto \exp \left[\left(D T_{0}\right) /\left(T-T_{0}\right)\right]$, where $T_{0}$ is the freezing temperature. A glass former is called fragile if $D$ is small, i.e., if the vicosity diverges rapidly as $T$ approaches $T_{0}$. Otherwise the glass former is "strong."

[30] G. Diezemann, J. Chem. Phys. 107, 10112 (1997); G. Diezemann, H. Sillescu, G. Hinze, and R. Böhmer, Phys. Rev. E 57, 4398 (1998).

[31] I.A. Campbell, J. Phys. (France) Lett. 46, L1159 (1985).

[32] I.A. Campbell, J.M. Flesselles, R. Jullien, and R. Botet, J. Phys. C 20, L47 (1987); N. Lemke and I.A. Campbell, Physica A 230, 554 (1996). 